\title{
Active substance applicator to remove the disturbances in the fluid flow
}

\author{
Joanna Grzegorczyn ${ }^{1}$, Jacek Trompeta ${ }^{2}$, Agnieszka Wróbel ${ }^{1}$, Krystian Kubica $^{3}$ \\ ${ }^{1}$ Student’s Scientific Group BioModel, Institute of Biomedical Engineering and Instrumentation, Wroclaw University of Technology, \\ Wroclaw, Poland \\ ${ }^{2}$ Oddział Neurochirurgii i Neurotraumatologii Wojewódzkiego Szpitala Specjalistycznego Nr 4, Bytom, Poland \\ ${ }^{3}$ Institute of Biomedical Engineering and Instrumentation, Wroclaw University of Technology, Wroclaw, Poland \\ Email: krystian.kubica@pwr.wroc.pl
}

Received 26 November 2013; revised 31 December 2013; accepted 12 January 2014

Copyright (c) 2014 Joanna Grzegorczyn et al. This is an open access article distributed under the Creative Commons Attribution License, which permits unrestricted use, distribution, and reproduction in any medium, provided the original work is properly cited. In accordance of the Creative Commons Attribution License all Copyrights @ 2014 are reserved for SCIRP and the owner of the intellectual property Joanna Grzegorczyn et al. All Copyright (c) 2014 are guarded by law and by SCIRP as a guardian.

\section{ABSTRACT}

The paper presents the construction of an automatic applicator of active substance that removes causes of the disturbances in the fluid flow in a system that is under the control. In a particular case, it can be applied to the removal of disturbances in the blood flow in vessels caused by a thrombus formation (e.g. postoperative or due to dialysis treatment). The presented applicator automatically releases the active substance into the system while detecting in real time the abnormal fluid flow. It allows mixing the active substance (e.g. anticoagulant drug) with the controlled medium outside the substance container (e.g. serum) while offering the possibility of the repeated usage.

\section{KEYWORDS}

\section{Drug Applicator; Thrombus; Dialysis; Fluid Flow}

\section{INTRODUCTION}

Blood circulation disorders are associated with cardiovascular disorders and diseases of civilization such as diabetes or obesity $[1,2]$. The direct causes of disruption in blood transport are due to structural deformations of vessels (e.g. mechanical wall damage, in atherosclerosis, calcification of the arterial walls, aneurysms or varicose veins), or due to disturbances in coagulation and fibrinolysis (increased internal vascular coagulation, fibrinolysis weakness and other disorders of the blood hemostasis). All of these increase the chances of the blood clot or thrombus (separate concepts) occurrence. Blood clots are formed as a result of blood coagulation when it leaves the damaged blood vessels or integuments. The latter emerges as a consequence of a local disturbance in coagulation and fibrinolysis homeostasis and its formation does not cause apparent symptoms until the thrombus starts interfering with the blood flow (it can move freely with the blood flow causing a blockage anywhere in the body).

Disturbances in the blood flow due to pathological vessels states or occurrence of bloods clots and thrombus are highly unfavorable. Blood clots and thrombus increase the risk of vascular occlusion and ischemia, which, subsequently when untreated, lead to tissue necrosis due to lack of blood (thus oxygen) supply, with the blockages of the blood flow in the brain vessels being particularly dangerous. The turbulences in the flow and the reduction of the blood flow velocity predispose the development of coagulation disorders. Endoaortic thrombosis can be observed in patients with primary hematologic disorders (in antiphospholipid syndrome, paraneoplastic coagulation disorder, aspergillosis or in long-term steroids treatment). In these cases, it is possible to observe the formation of the thrombus and nonadherent floating thrombus [3]. The positive diagnosis for the thrombus in artehrosclerosis (a condition in which an artery wall thickens narrowing its lumen as a consequence of the accumulation of cholesterol and other lipids activating the coagulation process) is a medical basis to perform angioplasty, a surgical widening the narrowed or obstructed arteries).

The identified risk factors for blood clotting occurrence as described by Virchow [4] include 1) damage of the blood vessel wall (mechanical trauma, burns, postphlebitic syndrome), 2) disturbed blood flow (atherosclerotic deposits, vasoconstriction), 3) changes in the composition of the blood (hypercoagulability syndrome, cancer, pregnancy). In diseases associated with these fac- 
tors, the occurrence of post-operative clotting time plays a key role for the administrated drugs to be effective. The probability of complications due to thrombus occurrence decreases with the correct timing of the appropriate drug administration directly into the bloodstream as compared to drugs administrated orally (in the form of tablets). However, the intravenously administrated anticoagulants require, on the other hand, trained personnel and the patient's careful monitoring in the intial administration phase (at later stages the monitoring frequency may be decreased based on the patients' response to the treatment).

The most commonly used anticoagulants include heparin, coumarin derivatives - vitamin $\mathrm{K}$ antagonists as well as a new type of drug acting as inhibitors of clotting factor Xa and thrombin inhibitors activating fibrinolysis. Thrombolytics are prescribed to dissolve blood clots that cause congestion by activating the endogenous clot dissolver system. However, the administration of the active substacne does not always give the desired results, in particular when plasmin (enzyme) cannot reach the location of the blockade due to blood flow disturbances. Therefore, ideally the drugs with this particular mode of action should be administrated locally at the place where the arterial obstruction occurs. The standard clinical practice is to administrate the drugs up to $12 \mathrm{~h}$ from the occurrence of pulmonary embolism, or myocardial infarction and up to $36 \mathrm{~h}$ in the case of vein thrombosis. These should not be offered to patients with high hypertension, peptic ulcer, or suffering from purpura (bleeding disorders) and thrombocytopenia. Another group of drugs is based on enzymes derived from snake venom and its mode of action is to reduce the amount of fibrinogen in the blood. Depending on the source origin, their administration yields various effects, e.g. ancrod [5] injected intravenously breaks down fibrinogen into smaller molecules that do not form crosslinks impeding the formation of blood clots and batroxobin disconnects fibrin monomer from fibrinogen with the resulting monomer activating plasminogen (injected intravenously, intramuscularly or subcutaneously) [6].

In most patients, the aforementioned treatments result in positive outcomes, however, in the case of excessive clotting in association with other diseases, the risk of complications remains high. Here, other techniques (mainly surgical) are used to remove the thrombi, e.g. during the procedure of embolectomy the blood vessel is opened and the material forming the blockage is removed and a removal of thrombus by fragmentation and aspiration by using a catheter (thrombectomy) may be a preferred choice in patients with contraindications to embolectomy. The thrombectomy and the aortas transplantation pose very high risk of complications, mainly from the cerebral circulation occurring in $29 \%$ of patients [3]. An inopera- ble method used in patients with pulmonary embolism is a temporal or permanent implantation of the filter into the inferior vena cava. This filter acts as a mechanical barrier capturing thrombus and preventing the formation of embolism [7].

Here, we present a schematical model of an applicator enabling an automated detection of the disturbances in the fluid (e.g. blood) flow with the simultaneous administration of the active substance (e.g. anticoagulant drug).

\section{MATERIALS}

The problem of the automatic release of the active substance into the bloodstream at the time of the detection of the disturbance in the blood flow in a section under control can be addressed via the usage of an applicator as shown in Figure 1.

The model of this applicator is made of two silicon pipes of an inner diameter of $6 \mathrm{~mm}$ each (labeled 2 and 3 on Figure 1) that share a common input (1) and a common output (7). The flow of liquid in these two ducts is automatically controlled by a connector (4) and a container (5) that encloses an active compound (here methylene blue, a blue dye) as well as including a vent. To restrict the free flow of the active compound the connector has the interconnected valves which consist of two pistons made of stearin (6). These valves separate the container from pipes 2 and 3 . The position of the pistons is controlled by a return spring (7). The suitable displacement of the pistons (valves) allows the flow of the solution of the active substance from the storage container through one of the connecting channels (8) to the corresponding pipe 2 or 3 . The fluid flow in the pipes (2) and (3) is subjected to the automatic control that is carried out by the connector (4), the container (5), the valves (6) and the connecting channels (8). A sudden reduction in the flow in one of the pipes (2 or 3), above or below the connector (4), results in a corresponding shift of the interconnected valves which in turn opens one of the connecting channels (8) and releases the active substance from the reservoir to the corresponding pipe (2 or 3). The removal of disturbance in the blood flow (eliminating the cause by the release of the actvie substance) in the controlled pipeline enables restoring the system to its original state via the spring (7). A connection of the two pipes (2) and (3) to the common output (9) enables the active subtance to enter to the whole system, where the circulation is forced by the pump.

\section{RESULTS AND DISCUSSION}

The construction of the applicator is analogous to the Wheatstone's bridge. Based on the Hagen-Poiseuille equation a vascular resistance can be defined as in Equation (1) 


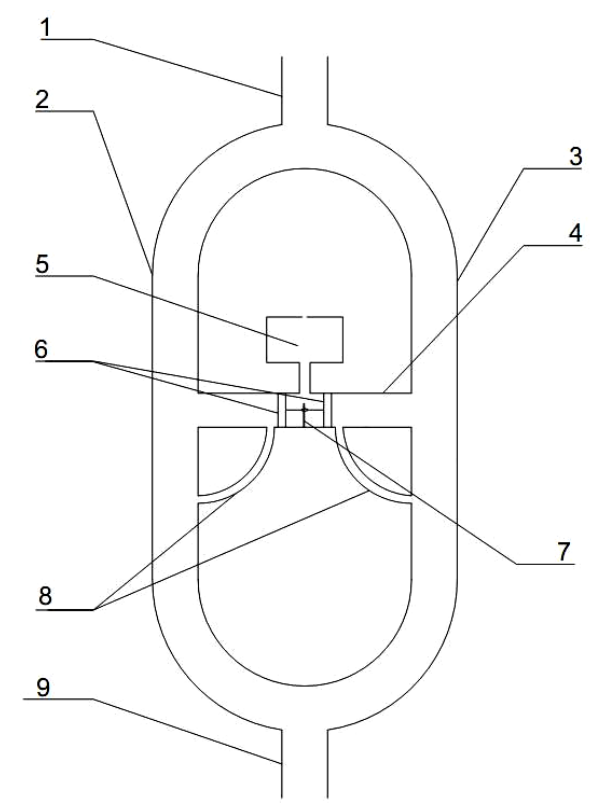

Figure 1. The applicator's scheme with (1) input, (2) and (3) pipes, (4) connector, (5) container, (6) pistons, (7) spring, (8) connecting channel and (9) output.

$$
R_{f}=\frac{8 \eta l}{\pi r^{4}}
$$

where:

$R_{f}$ is the vascular resistance;

$\eta$ is the dynamic fluid viscosity;

$r$ is the internal radius of the tube;

$l$ is the length of the tube.

By analogy to bridge the relationship between the respective pressure differences can be expressed by the Equation 2:

$$
\Delta p_{C D}=\Delta p_{A B}\left(\frac{R_{f 1}}{R_{f 1}+R_{f 3}}-\frac{R_{f 2}}{R_{f 2}+R_{f 4}}\right)
$$

where:

$R_{f 1}, R_{f 2}, R_{f 3}, R_{f 4}$-are resistances of pipes 1 and 2 (above and below the connector 4 , Figure 1 );

$\Delta p_{C D}$ is the pressure difference at both ends of the connector;

$\Delta p_{A B}$ is the pressure difference between the inlet and outlet.

An increase in the resistance $R_{f 1}$ or $R_{f 4}$ leads to a maximum $\Delta p_{C D}$ change equal to $0.5 \Delta p_{A B}$ while an increase in the resistance $R_{f 2}$ or $R_{f 3}$ leads to a maximum $\Delta p_{C D}$ change equal to $-0.5 \Delta p_{A B}$.

The presented applicator automatically releases the desired substance into the controlled circulation at the time of the occurrence of the disturbance in the fluid flow. The active substance (marked with methylene blue dye) is automatically released into the water flow when the blockage appeares in the pipe segment being under control, in particular drugs removing thrombus or extending blood vessels in the bloodstream.

In the studied system the flow of water $\left(180 \mathrm{ml} \cdot \mathrm{min}^{-1}\right)$ is divided into two streams (Figure 2(a)). In the circulatory system this division may be done on the basis of anatomical distribution, or by introducing additional artificial reference pipe. The drug located in the cartridge is released into the blood as the differential pressure between the two pipes appears. Figure 2 shows the release of a solution (with blue dye, representing the active substance) which is induced by a reduction in a flow of fluid in the controlled pipe. The flow disturbance was modeled by placing a clip above (or below) the connector of the two pipes. Placing the clip on the left pipe below the connector moves the pistons to the right which in turn leads to release the dye solution to the right pipe (Figure 2(b)). A reduced fluid flow in the left pipe above the connector yields the movement of the pistons to the left and the dye solution can escape from the container to the same pipe (Figure 2(c)). The system behaves analogously when there are flow disturbances in the right pipe above and below the connector (Figures 2(d) and (e)).

The considered solution simultaneously detects the disturbances in the fluid flow and reacts with the release of a potentially active substance can find numerous applications to the circulatory system where the blood flow is disturbed due to pathological occurrence of blood clots and thrombus. For instance, it can aid controlling flow of blood in the dialysis tubing.

Figure 3 shows how the applicator can control the dialysis process, with this solution being of significance when the main and/or the emergency power system fails. Aretriovenous dialysis can be then performed with the Mariotte bottle in the dialysate circuit. In the event of the thrombus formation in the dialyser column (despite of the addition of heparin), the applicator automatically releases clot-dissolving substances into the bloodstream, protecting patient's circulatory system.

The described applicator could reduce the amount of substances that prevents blood clotting in the dialysis circuit. An additional quantity of the drug would be added at the time of appearance of the abnormal blood flow. Another potential application is in the control of blood flow disturbances during the postoperative period, especially when the vessels need to merge increasing the risk of blood clots. In this case, one of the arms (pipes) of the applicator is made up of a natural blood vessel, while the second arm (pipe) can be of an artificial biocompatible material. Increasing the cross-section of the artificial pipe reduces the risk of the blood flow disturbances. However, changing the diameter of the pipe requires proper matching the diameter of the two connected pistons (valves) so not to allow the release of the active 


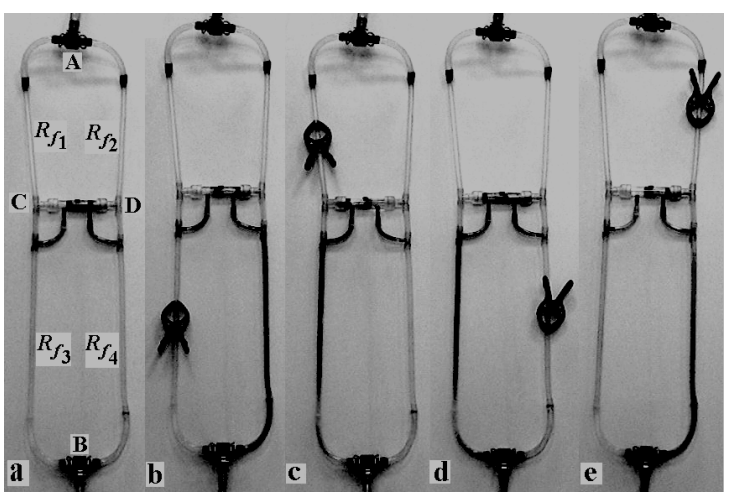

Figure 2. The applicator with (a) no disturbance in the fluid flow (the flow in neither of the two arms is disturbed; $R f_{1}, R f_{2}, R f_{3}$ and $R f_{4}$ - pipe resistances; (b) the flow disturbed in the left pipe below the connector with storage of active substance; (c) the flow disturbed above the connector; (d) the flow disturbed in the right pipe below the connector; (e) the flow disturbed in right pipe above the connector.

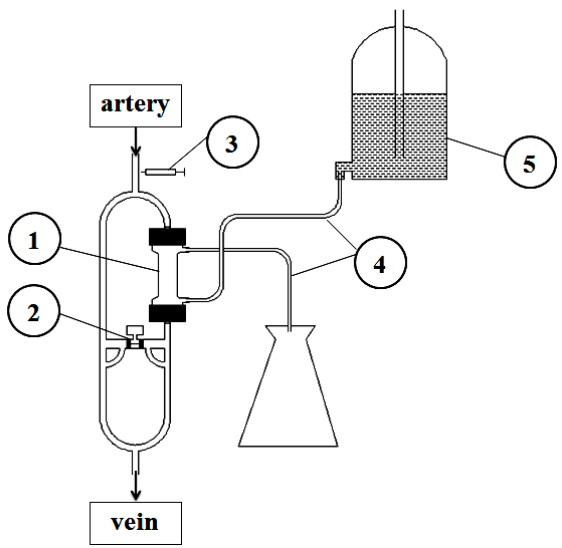

Figure 3. Figure shows how the applicator can contol the dialysis process, with this solution being of significance when the main and/or the emergency power system fails. (1) dialyser, (2) applicator of thrombolytics, (3) heparin pump, (4) dialysate line, (5) Mariotte’s bottle.

substance in the absence of the flow disturbance.

The major difficulty in the construction of the applicator is the selection of materials, which all need to be blood compatible. Currently there are no existing materials that used exclusively and long-term would be risk free in terms of causing blood clots. There are many studies to improve biocompatibility, e.g. the materials with a modified surface, covered with a thin film of synthetic polymer or a layer of gelled material to prevent the aggregation of thrombocytes have been tested [8]. Currently, the most commonly used materials comprise of linear, saturated polymers such as poly (ethylene) terephthalate and poly tetrafluoroethylene. Typically, vascular prostheses are made in the form of knitted fabric, less often textiles [8]. An important aspect of the optimization is the selection of the geometry of connections in order to compensate for turbulence, which could reduce the likelihood of thrombus formation in this area.

\section{CONCLUDING REMARKS}

The presented applicator can be used in automatic control of flows in a closed circuit, in the particular case of blood circulation during dialysis or temporarily in the postoperative period, when the risk of blood clots is increased.

\section{REFERENCES}

[1] Fox, C.S. and Coady, S. (2007) Increasing cardiovascular disease burden due to diabetes mellitus: The Framingham heart study. Circulation, 115, 1544-1550.

http://dx.doi.org/10.1161/CIRCULATIONAHA.106.6589 $\underline{48}$

[2] Ku, D. (1997) Blood flow in arteries. Annual Review of Fluid Mechanics, 29, 399-434. http://dx.doi.org/10.1146/annurev.fluid.29.1.399

[3] Rancic, Z., Pfammatter, T., et al. (2009) Floating aortic arch thrombus involving the supraaortic trunks: Successful treatment with supra-aortic debranching and antegrade endograft implantation. Journal of Vascular Surgery, 50, 1177-1180. http://dx.doi.org/10.1016/j.jvs.2009.05.052

[4] Bagot, C. and Arya, R. (2008) Virchow and his triad: A question of attribution. British Journal of Haematology, 143, 180-190. http://dx.doi.org/10.1111/j.1365-2141.2008.07323.x

[5] Pizzo, S.V., Schwartz, M.L., Hill, R.L. and McKee, P.A. (1972) Mechanism of ancrod anticoagulation. A direct proteolytic effect on fibrin. Journal of Clinical Investment, 51, 2841-2850. http://dx.doi.org/10.1172/JCI107107

[6] Castroa, H.C., Zingali, R.B., Albuquerquec, M.G., PujolLuza, M. and Rodriguesd, C.R. (2004) Snake venom thrombin-like enzymes: From reptilase to now. Cellular and Molecular Life Sciences, 61, 843-856. http://dx.doi.org/10.1007/s00018-003-3325-z

[7] http://www.radiologyinfo.org/en/info.cfm?pg=venacavafi lter

[8] Chlupác, J., Filová, E. and Bacáková, L. (2009) Blood vessel replacement: 50 years of development and tissue engineering paradigms in vascular surgery. Physiological Research, 58, 119-139. 